\title{
Conservation of the Segmented Germband Stage: Robustness or Pleiotropy?
}

Frietson Galis (galis@rulsfb.leidenuniv.nl)

Tom J.M. van Dooren (vdooren@rulsfb.leidenuniv.nl)

Johan A.J. Metz (metz@rulsfb.leidenuniv.nl)

\section{Approved by}

Ulf Dieckmann (dieckmann@iiasa.ac.at)

Project Leader, Adaptive Dynamics Network

June 2002 


\section{Contents}

Effects of Mutations Acting on the Extended and Segmented Germband Stage ............. 3

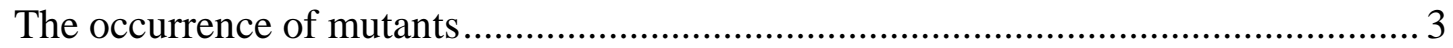

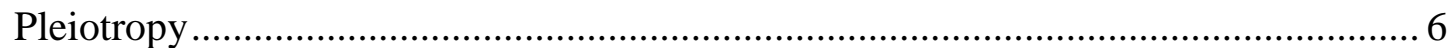

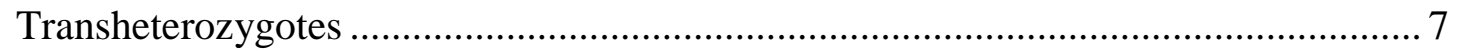

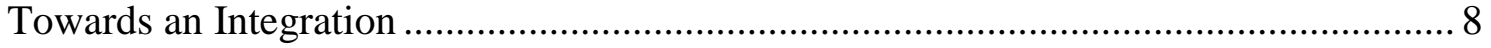

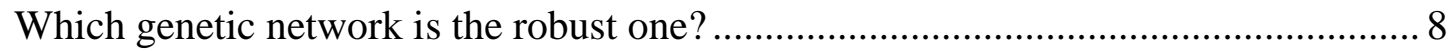

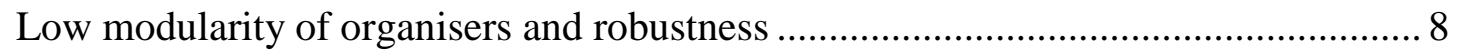

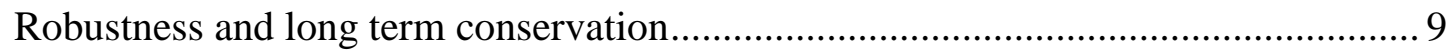

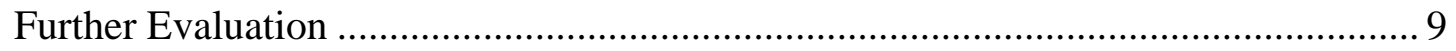

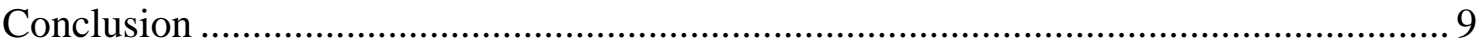

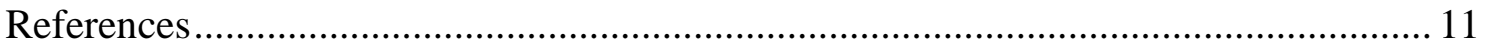




\begin{abstract}
Gene expression patterns of the segment polarity genes in the extended and segmented germband stage are remarkably conserved among insects. To explain the conservation of these stages two hypotheses have been proposed in the literature. One hypothesis states that the conservation is due to a high interactivity between modules so that mutations would have multiple pleiotropic effects in other parts of the body, resulting in stabilizing selection against mutational variation. The other hypothesis states that the conservation is due to robustness of the segment polarity network against mutational changes. When evaluating the empirical evidence for these hypotheses, we found strong support for pleiotropy and little evidence supporting robustness of the segment polarity network. This points to a key role for stabilizing selection in the conservation of these stages. Finally, we discuss the implications for robustness of organizers and long term conservation in general.
\end{abstract}




\title{
About the Authors
}

\author{
Frietson Galis \\ Institute of Evolutionary and Ecological Sciences \\ Leiden University \\ P.O. Box 9516 \\ 2300 RA Leiden \\ The Netherlands \\ Tom J.M. van Dooren \\ Institute of Evolutionary and Ecological Sciences \\ Leiden University \\ P.O. Box 9516 \\ 2300 RA Leiden \\ The Netherlands \\ Johan A.J. Metz \\ Institute of Evolutionary and Ecological Sciences \\ Leiden University \\ P.O. Box 9516 \\ 2300 RA Leiden \\ The Netherlands \\ and \\ Adaptive Dynamics Network \\ International Institute for Applied Systems Analysis \\ A-2361 Laxenburg, Austria
}

\section{Acknowledgments}

We thank Ricardo Azevedo for insightful discussions. We thank Jacques van Alphen, Elizabeth van Ast, Ricardo Azevedo and Patricia Beldade for comments on the manuscript. 


\title{
Conservation of the Segmented Germband Stage: Robustness or Pleiotropy?
}

\author{
Frietson Galis \\ Tom J.M. van Dooren \\ Johan A.J. Metz
}

Morphogenetic patterning during early embryonic stages, including the process of segmentation, has diverged markedly among insects. The diversity in morphogenetic patterning is reflected in the diverse expression patterns of the genes involved (gap and pair-rule genes, 1-5). However, at the end of gastrulation, developmental trajectories converge towards a highly conserved stage coinciding with early organogenesis: the segmented germband stage (Fig. 1; 2,5-8). Conservation of morphological patterns and expression of genes correspond: both the striped expression patterns of the segment polarity genes and the co-linear pattern of the Hox genes are remarkably conserved (24,9).

Sander (7) and Raff (10) hypothesize that high connectivity between modules (Fig. 2 ) is the major cause of conservation in the segmented germband stage. This high connectivity causes mutational changes to have multiple pleiotropic effects that become amplified as development proceeds. Since pleiotropic effects during embryogenesis are generally disadvantageous $(11,12)$, strong stabilizing selection against mutational variation ensues. In this scenario, conservation is due to consistently strong selection against mutations via their pleiotropic effects. Strong connectedness of modules implies an easily destabilized network of inductive events, with low effective robustness and low effective modularity (Fig. 2). Although the hypothesis was proposed for the conservation of the segmented germband stage, it is natural to include the earlier extended germband stage (Fig. 1) when evaluating its explanatory power, as the characteristic gene expression patterns of the segment polarity and Hox genes are then already present.

Von Dassow and Munroe (13) also assume that conservation is due to network characteristics. They hypothesize that the network of the segment polarity genes is causally involved in the conservation of the expression pattern of the segment polarity genes in the ectoderm while referring to Von Dassow's et al.'s (14) model on the robustness of that network. In robust gene networks, by definition, developmental noise and mutations do not lead to clear phenotypic effects because gene interactions tend to neutralize perturbations and in particular make mutations recessive $(15,16)$. According to Von Dassow et al. (14), robustness should buffer the network both against changes of the input at the start of the network (i.e., changes in the signals from the preceding stage) and against changes in the input during its running. Together, the two articles 


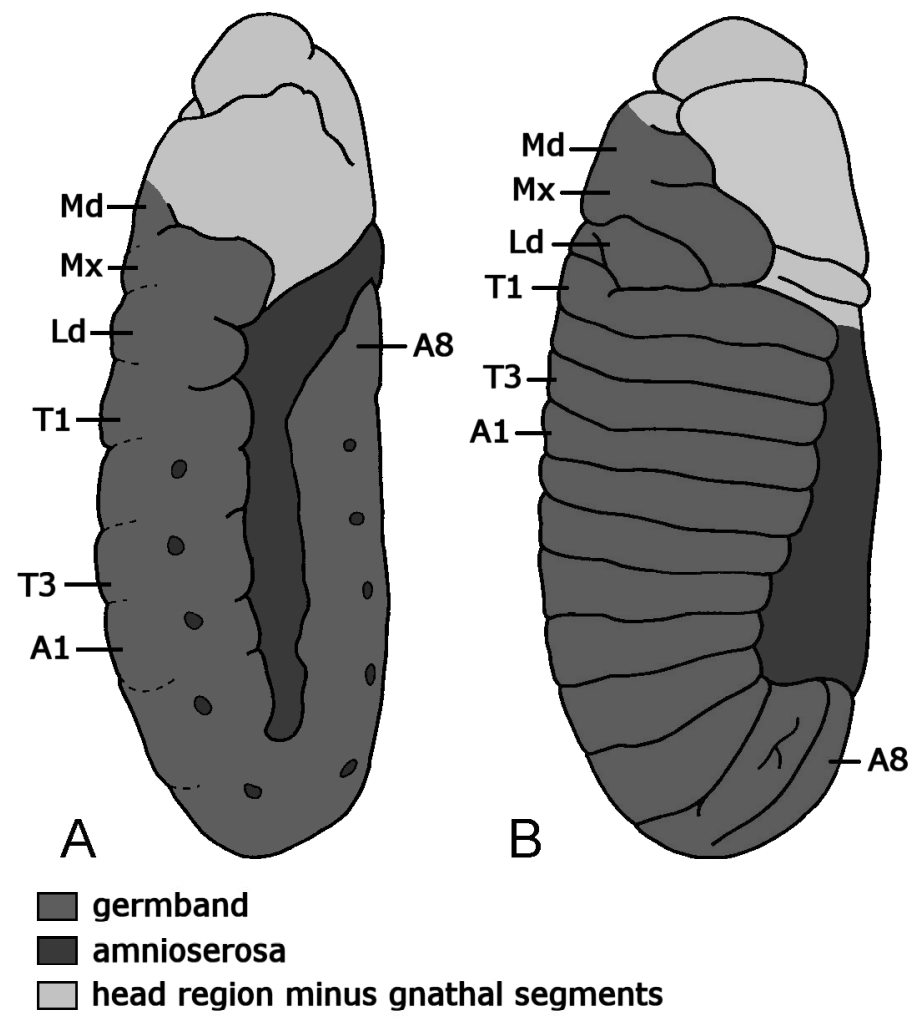

F ig u re 1. Schematic figure of extended (A) and segmented germband stage (B) in Drosophila. The germband (blue) refers to the part of the embryo that will give rise to the metameric regions of it: gnathal segments (Ma, mandible, Mx, maxilla, Lb, labium), thoracic segments, $\mathrm{T}$ and abdominal segments, A). The amnioserosa (red) is an extra-embryonic membrane. The extended germband stage starts approx. 6.5 hours after fertilization and the segmented germband stage ends at approx. 10.5 hours after fertilization.

suggest that conservation of the network occurs despite accumulation of genetic changes, because these changes have little phenotypic effect and mainly lead to hidden variation.

The robustness hypothesis was only proposed for the segment polarity gene network involved in the striped expression pattern occurring in the epidermis during the extended and segmented germband stage (Table 1). Von Dassow et al. (14) are the first to model this network and its robustness, which is very valuable. However, the model needs to be evaluated with respect to influences by genes directly regulating the network. If the network is robust to changes in its input, mutations in genes regulating the genes in the network should not affect its activity, e.g. eve, ftz, slp, tsh, Dpresenilin, hid and genes of the Notch pathway. Otherwise, the network is not robust in reality, with the predicted robustness hinging on the specific modularity assumptions made by von Dassow et al.

During the extended and segmented germband stages the segment polarity network acts as an organizer central to many patterning events (Box 1). These stages are conserved as a whole. Therefore, the robustness hypothesis should be extended to the organization of those stages in full, otherwise it can never explain evolutionary conservation.

The two hypotheses become diametrically opposed when extended to the overall conservation of the two stages. The pleiotropy hypothesis points to a low modularity as the cause of the conservation, whereas the robustness hypothesis assumes a high 
I a b le 1. Constituents of the segment polarity gene network modeled by Von Dassow et al. (2000).

\begin{tabular}{|l|l|}
\hline $\mathrm{Wg}$ & wingless \\
\hline $\mathrm{Cn}$ & repressor fragment of $\mathrm{CiD}$ \\
\hline $\mathrm{En}$ & engrailed \\
\hline $\mathrm{Ptc}$ & patched \\
\hline $\mathrm{Hh}$ & hedgehog \\
\hline $\mathrm{Ph}$ & patched-hedgehog complex \\
\hline $\mathrm{CiD}$ & cubitus interruptus Dominant \\
\hline
\end{tabular}

I a ble 2. Predictions of the extended robustness and pleiotropy hypotheses.

\begin{tabular}{|l|l|l|}
\hline \multicolumn{2}{|c|}{ Effect of mutations } \\
\hline & Pleiotropy hypothesis & Robustness hypothesies \\
\hline $\begin{array}{l}\text { genetic mututational } \\
\text { variation }\end{array}$ & $\begin{array}{l}\text { visible at the phenotypic } \\
\text { level }\end{array}$ & hidden \\
\hline direct phenotypic effects & potentially large & small \\
\hline $\begin{array}{l}\text { dominance of direct } \\
\text { effects }\end{array}$ & $\begin{array}{l}\text { haplo-insufficiency } \\
\text { possible }\end{array}$ & $\begin{array}{l}\text { recessivity or near } \\
\text { recessivity }\end{array}$ \\
\hline pleiotropic effects & many & few \\
\hline
\end{tabular}

modularity for inductive interactions between segments and germ layers as the cause (fig.2). Therefore, they imply strikingly different roles for modularity in evolution: constraining versus facilitating evolutionary change. Furthermore, they lead to different predictions regarding mutations affecting the segment polarity gene activity in the extended and segmented germband stage (Table 2). Below, we test the explanatory power of the robustness and pleiotropy hypothesis for the overall conservation of these stages, while placing special emphasis on the segment polarity gene network in the ectoderm.

\section{Effects of Mutations Acting on the Extended and Segmented Germband Stage}

\section{The occurrence of mutants}

Mutant screens with sensitized genetic backgrounds have probably uncovered the majority of genes affecting segmentation. For instance, Müller et al.(17) carried out a translocation screen for zygotically expressed genes in Drosophila that covered more than $99 \%$ of the genome. They found that nearly all zygotically expressed genes that regulate $w g$ expression in the ectoderm had already been identified previously. Moreover, nearly all these documented mutations appear to have a phenotypic effect. 
(A) Robustness
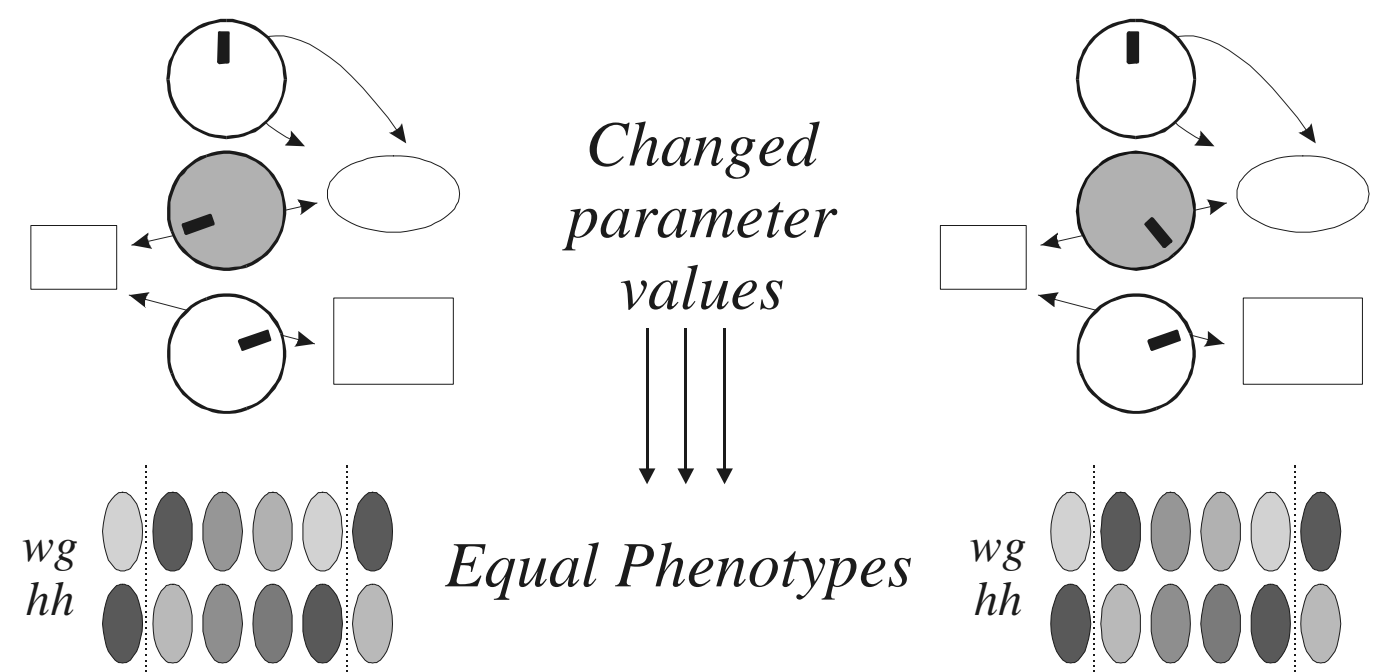

\title{
(B) Interacting M o d ules
}

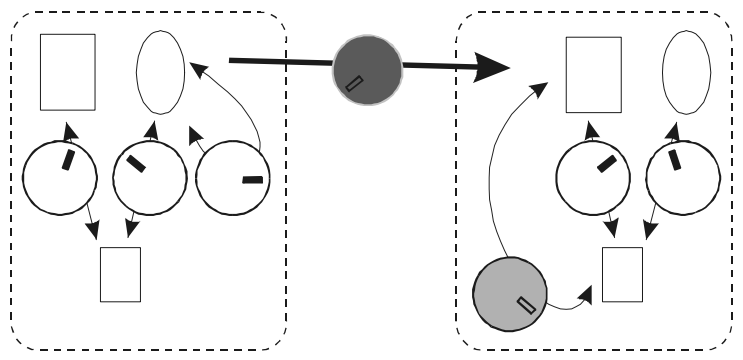

\author{
Low Connectivity \\ $=$ \\ High Effective Modularity
}

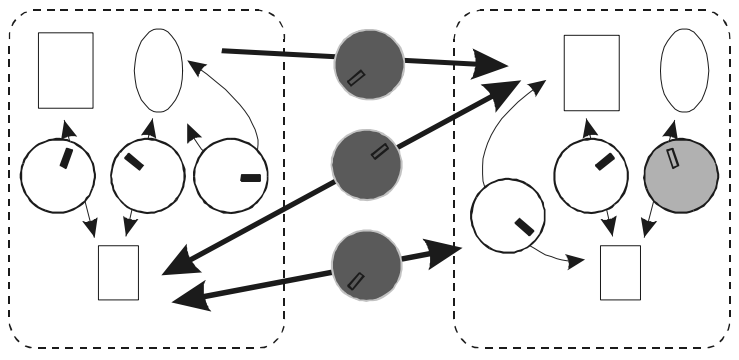

\author{
High Connectivity \\ - \\ Low Effective Modularity
}

F igure 2. Schematic figure explaining (A) robustness and (B) effective modularity. (A) When a parameter is changed in a robust genetic network, the resulting phenotype does not change (in this case illustrated with the concentration of wingless (wg) and hedgehog (hh) in the cells of the ectoderm). (B) Modules are discernible and discrete units within large genetic networks that have some autonomy and a clear physical location (10). They can differ in the amount of connectedness. First of all, all input to robust elements of a module can be ignored, since it will have no discernible effect. A large proportion of robust components in a module therefore reduce potential connectivity. Low connectivity, with few connections having small effects, implies high effective modularity. High connectivity implies low effective modularity. 
B os 1. C ascading pleiotropic effects

The signaling of the segment polarity genes in the ectoderm acts as an organizer affecting many processes, e.g., the segregation and early differentiation of neuroblasts, epidermoblasts, sensory precursor cells, salivary precursor cells, imaginal discs and tracheal precursor cells in the ectoderm (ac). The downstream effects involve a cascade of signaling across segmental and germ layer boundaries. For instance, the subdivision of the mesoderm into the primordia of heart, fat body and visceral mesoderm, as well as the differentiation of the somatic mesoderm, are regulated by segment polarity genes with signals coming from both ectoderm and mesoderm (d-f). Signaling from the mesoderm in turn is crucial for the local differentiation of the non-segmented endoderm and ectodermal gut (f-h). Signaling from the gut influences the patterning of the visceral mesoderm (g,i). Patterning of the visceral mesoderm also involves signaling across segmental borders, e.g. for the anterior migration of caudal precursor cells of the longitudinal musculature around the gut. Any abnormality in the trunk visceral mesoderm disrupts this migration (j). The migration of somatic muscle precursors also involves very complex signaling between germlayers, with a key role for the activity of segment polarity genes around the parasegmental border in the ectoderm $(k, l)$. Signaling across germline and segmental borders must also be involved in the differentiation and migration of the nervous system and trachaeae. The complex branching pattern of the tracheal network is established by migrating precursors (m). Again, segment polarity genes, especially $h$, appear to play a central role (n). Furthermore, signaling between the ectoderm and the extra-embryonal layer (amnioserosa) is crucial for two essential morphogenetic processes, to wit, germband retraction and dorsal closure $(o, p)$. The $w g$ pathway influences this patterning in an interaction with the JnK pathway (p). Finally, cell death probably plays an important role in the cascade of pleiotropic effects. Programmed cell death occurs at a relatively high rate during the extended and segmented germband stages and activity of the segment polarity genes (in particular $W g$ ) plays an important role in the patterning of cell death (q).

\section{References}

a. Lawrence, P.A. et al. (1999) The Hedgehog morphogen and gradients of cell affinity in the abdomen of Drosophila. Development 126, 2441-2449

b. Hatini, V. and Dinardo, S. (2001) Divide and conquer: pattern formation in Drosophila embryonic epidermis 17, 574-579

c. Michelli, C.A. et al. (2002) rasp, a putative transmembrane acyltransferase, is required for Hedgehog signaling. Development 129, 843-851

d. $\quad$ Azpiazu N. et al. (1996). Segmentation and specification of the Drosophila mesoderm. Genes Dev. 10: 3183-94

e. Riechmann, V. et al. (1997) Control of cell fates and segmentation in the Drosophila mesoderm. Development 124, 29152922

f. Lee, H.-H. and Frasch, M. (2000) Wingless effects mesoderm patterning and ectoderm segmentation events via induction of its downstream target sloppy paired. Development 127, 5497-5508

g. Hoch, M. and Pankratz, M.J. (1996) Control of gut development by fork head and cell signaling molecules in Drosophila. Mech. Devel. 58, 3-14

h. Bienz, M. (1997) Endoderm induction in Drosophila: the nuclear targets of the inducing signals. Curr. Opinion Genet. Devel. 7, 683-688

i. Martin B.S. and Bate, M. (2001) Hindgut visceral mesoderm requires an ectodermal template for normal development in Drosophila. Development 128, 233-242

j. Zaffran, S., Kuchler, A., Lee, H.-H. and Frasch, M. (2001) biniou (FoxF), a central component in a regulatory network controlling visceral mesoderm development and midgut morphogenesis in Drosophila. Genes Dev. 15, 2900-2915

k. Volk, T., Vijay Raghavan, K (1994) The central role for epidermal segment obrder cells in the induction of muscle patterning in the Drosophila embryo. Development 120, 59-70

1. Baehrecke, E.H. (1997). Who encodes a KH RNA binding protein that functions in muscle development. Development $124,1323-1332$

m. Hartenstein, V. and Y. N. Jan (1992) Studying Drosophila embryogenesis with P-lacZ enhancer trap lines. Roux's Arch. Dev. Biol. 201:194-220

n. Glazer,L.and Shilo, B.-Z. (2001) Hedgehog signaling patterns the tracheal branches. Development 128, 1599-1606

o. Lamka, M.L., Lipshitz, H.D. (1999) Role of the amnioserosa in germ band retraction of the Drosophila melanogaster embryo. Devel. Biol. 214, 102-112

p. McEwen, D.G., Cox, R.T. and Peifer, M. (2000) The canonical Wg and JNK signaling cascades collaborate to promote both dorsal closure and ventral patterning. Development 127, 3607-3617

q. Cox, R.T., D.G. McEwen, D.L. Myster, R.J. Duronio, J. Loureiro, Peifer, M. (2000) A screen for mutations that suppress the phenotype of Drosophila armadillo, the $\beta$-catenin homolog. Genetics 155, 1725-1740 
Spontaneous mutations with a phenotypic effect on the extended and segmented germband stages have long been documented. Especially mutations that cause homeotic changes have received attention (18). Later, other spontaneous mutations with an effect during these stages were recovered in laboratories: mutants of the engrailed (19) and Cubitus interruptus (20) genes modelled by Von Dassow, and mutants of other genes $(21,22)$.

There is an abundance of induced mutations with an effect on the extended and segmented germband stages, including an effect on the segment polarity network in the ectoderm. A number of these mutations change the initial input of the segment polarity gene network in the ectoderm and this usually leads to severe abnormalities in the striped segment polarity gene expression (e.g., mutations of the pair rule genes, 17, 2326). In addition, many mutations affect the input during the active phase of the network in the ectoderm and have similarly drastic abnormalities as a result (including mutations of the Notch and JNK pathway, e.g. 23,27-32). For a few mutations no or few phenotypic effects are reported, due to overlapping gene functions. This occurs in invectedlengrailed, $f z / d f z(2)$ and cubitus interruptus/teashirt mutations $(17,34,35)$. The robustness provided by gene redundancy differs from that considered in von Dassow et al's model.

One can argue that most of these mutants correspond to loss of function and thus fall out of the scope of the robustness modeled by Von Dassow (14). However, severe effects are also observed in hypomorphic mutations that only reduce gene functions. Many hypomorphic mutations disrupt the normal signaling of segment polarity genes in the ectoderm and elsewhere, often with lethal phenotypic effects (fig. 3 and 4, e.g. en, $w g$, ptc, arm, dsh, porc, eve; 24,25,27, 36-40). Comparisons of hypomorphic and other mutants show that cells are sensitive in their response to different concentrations of $w g$ (e.g., in ectoderm and imaginal discs, $23,27,41)$ and $h h(42,43)$. This lack of robustness ensures that throughout the embryo small differences in gene dosage can lead to severe effects. In addition cell responses appear sensitive to dosages of other segment polarity genes and genes affecting their expression ( $g s b$, arm, ptc and $n k d$, hid; 23,36,44,45). The changed expression patterns of segment polarity genes usually maintain a striped character in a segmentally iterated fashion. However, changes in the position, shape and intensity of stripes lead to dramatic phenotypic effects, so that the system does not appear robust.

Although most mutations affecting the extended and segmented germband stages are indeed nearly recessive, dominant mutations occur. Examples are Cubitus interruptus (20), fused (segment polarity gene), krüppel, lethal myospheroid, notch, delta, deformed (12) and Antp (46). Also, most recessive lethal mutations are not innocuous in heterozygous condition (11). In most investigated cases they are associated with a reduced viability and a changed developmental rate.

\section{Pleiotropy}

Although some robustness may occur, most mutations affecting the extended and segmented germband stage have dramatic pleiotropic effects. Mutations of segment polarity and other genes produce disturbances in many parts of the embryo $(9,11,12,29,40,46,47)$. Interestingly, pleiotropic effects are also found for hypomorphic mutations, in the ectoderm and elsewhere. For instance, hypomorphic $w g$ mutants have besides a disturbed striped segment polarity expression, defects in thorax, antennae, 


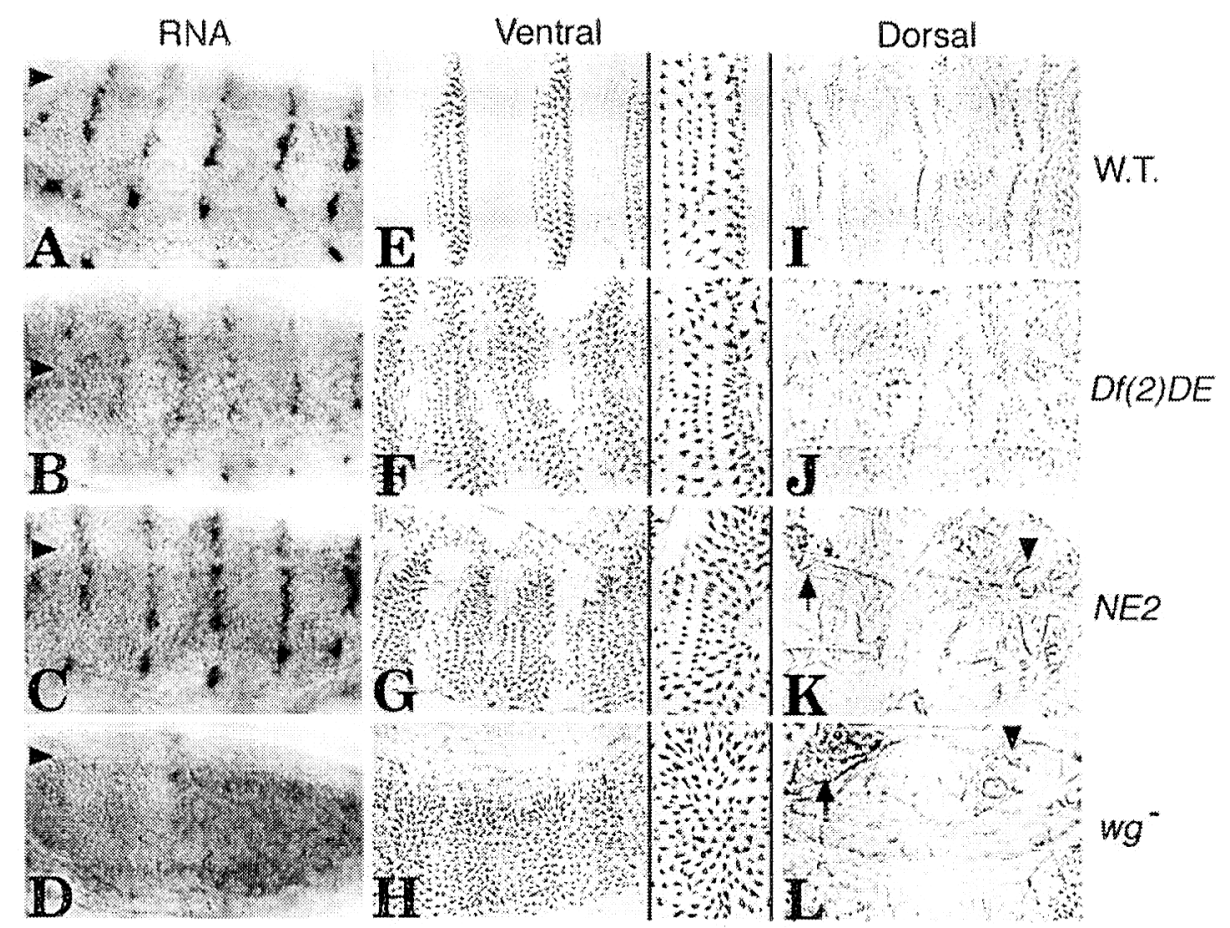

F ig u r e 3. Null (wg-), Reduced (Df(2)DE and partial (NE2) function mutations of the $w g$ gene lead to abnormalities in the larval ectoderm. Expression of $w g$ in the ectoderm (A to D), cuticular pattern in the ventral ( $\mathrm{E}$ to $\mathrm{H}$ ) and dorsal (I to L) larval epidermis (W.T. is wildtype). In $\operatorname{Df}(2) \mathrm{DE}$ mutants $w g$ expression is reduced, in NE2 wg transport is hampered (from Dierick and Bejsovec, 1998).

wings and in polarity in general (fig. $4 ; 27,39,48$ ). It is not surprising that pleiotropy is widespread. The segment polarity genes are, together with Hox and other genes, involved in many functions during this stage: the specification and early differentiation of virtually all organ primordia and the patterning of drastic morphogenetic events (e.g. germband retraction, dorsal closure and head involution). In addition, changes in the activity of these genes have important pleiotropic effects because of downstream cascading effects, especially related to the organizer activity of the segment polarity genes in the ectoderm (Box 1). The overall picture agrees with the high connectivity that underlies the pleiotropy hypothesis. The downstream cascading effects show that the interactions across compartment boundaries are considerable and that the overall level of effective modularity is, therefore, low.

\section{Transheterozygotes}

Pleiotropic effects of mutations in the conserved early embryonic stages will no doubt lead to severe and rapid selection against most mutations. Selection is usually expected to be very slow against recessive mutations. However, the picture changes if we also account for haplo-insufficiency when different recessive mutations on the same locus are combined. Kornberg (47) found that for 58 mutations at the engrailed locus virtually all combinations of two mutations in heterozygous condition (transheterozygotes) were lethal and all combinations had phenotypic effects. For wg, Gsb, and Antp similar transheterozygous effects were found (e.g. 24,44,46). In addition, many mutations show 




F ig u r e 4. Hypomorphic $\mathrm{Wg}^{1}$ mutant showing a failure in the development of antennae, wings, halteres and thorax (half of it missing, scutellum missing and hairs deranged (from Sharma and Chopra 1976).

severe and usually lethal haplo-insufficient effects in combination with mutations of different genes in the same or interacting pathways (e.g. hh, 43; wg, dsh 24; ptc, nkd 23; dTCF, puckered, hid and Dpresenilin, 45). This combined haplo-insufficiency will severely constrain the accumulation of recessive mutations.

\section{Towards an Integration}

\section{Which genetic network is the robust one?}

We agree with Von Dassow et al. (14) that robustness could play an important evolutionary role in allowing evolvability of stages preceding the conserved stages. The evolutionary diversification among insects of the gap and pair rule genes would indeed have been facilitated by robustness of the network that patterns the conserved stripes of $w g$ and $e n / h h$ signaling cells. However, the appearance of the segment polarity stripes precedes the signaling network as modeled by Von Dassow et al (14). In the initial phase of the conserved striped pattern $w g$ is not yet dependent on en and involves activity of pair rule genes (17). It is possible that robustness resides in this earlier more evolutionarily diversified signaling network, allowing evolutionary change of the preceding phase. Otherwise, it is difficult to indicate any particular phase of the gene network that could be characterized as robust. The signaling of the segment polarity genes during the time of the striped expression seems to be in a state of flux: e.g. after the initial phase en expression first depends on $w g$ expression in the ectoderm, then en and $w g$ mutually require each other's expression, yet later $e n$ is no longer dependent on $w g$ and finally both are independent of each other $(17,23,25,49,50)$. Adding to the dynamics are the many spatial differences in expression, e.g. the interaction of the segment polarity genes differs in the dorsal and ventral ectoderm $(23,27,51)$ and in the mid-lateral gap that appears in the $w g$-expressing stripe in the epidermis (49). The upstream and downstream interactions of the segment polarity genes, thus, show a surprisingly dynamic pattern in space and time.

\section{Low modularity of organisers and robustness}

Despite the observed dynamic pattern, we agree with Von Dassow et al. (14) that the input of the segment polarity gene network should be as robust as possible to changes. Yet, we expect that small changes in the connectivity of the segment polarity genes will have major effects on the outcome. The $w g$ and en/hh signaling cells along the parasegmental boundary function as an organizer (Box 1). In organizers small changes in output cause a cascade of effects because they organize a large part of the patterning 
in embryos. This also holds for any organizer during the earlier evolutionarily more diversified stages of cleavage and gastrulation. Therefore, organizers are not independent modules as they affect many processes in different parts of the embryo. The chance of a feedback effect on the input of the organizer is for this reason considerable, further lowering independence of modules and, in addition, affecting robustness.

\section{Robustness and long term conservation}

Stabilizing selection is expected to lead to robustness to protect optimized traits against developmental noise and mutations (16,52-55). This robustness can produce short-term conservation. However, during periods with drastic environmental changes that lead to strong directional selection, the robustness of genetic interactions can never be sufficiently high to prevent change. A robust gene network is characterized by selective neutrality of mutants, causing genetic drift within the set of morphogenetically equivalent genotypes and, thus, the accumulation of hidden variation (55). When there is selection for robustness, the neutral set is expected to grow larger. However, after a while, through combined effects of drift and constraints on the maximum achievable robustness, mutations come within reach that lead to a loss of robustness (the genetic composition of the population reaches the boundary of the neutral set, 55). This process is facilitated by the fact that neutral sets in genotypic sequence spaces tend to have large boundaries relative to their interior $(56,57)$, so that the proportion of genotypes close to the boundary is large.

\section{Further Evaluation}

The next step in investigating the support for both hypotheses should be a comparison between the segmented germband stage with earlier or later stages, where the vulnerability to mutations and the amount of genotypic variation without phenotypic effects are assessed. We recently analysed teratological studies in vertebrates for reported phenocopies of mutational change during other developmental stages and the pharyngula stage (59), the vertebrate phylotypic stage comparable to the germband stage in insects. Our study supports the validity of Sander's (7) and Raff's (10) hypotheses for the conservation of the vertebrate phylotypic stage. If a similar pattern would turn up in insects, this would further underpin the important role of pleiotropy and stabilizing selection in evolutionary conservation.

\section{Conclusion}

We found little evidence for robustness of gene networks towards mutational change acting on the extended and segmented germband stages, and more specifically the segment polarity gene network in the ectoderm. The phenotypic effects of even weakly hypomorphic mutations are in agreement with the observation of Lande et al. (58) that mutations of small, nearly additive effects are usually expressed relatively late in development, whereas lethal mutations are usually expressed early (see also 11,12). The organizer function of segment polarity and other genes causes mutations in these genes to have a cascade of pleiotropic effects. In addition, many auto-regulatory and crossregulatory interactions provide feedback on the input of the segment polarity gene network. As a result the segment polarity gene network shows relatively low effective 
modularity and robustness. The feedback that modulates the input of the network is absent in Von Dassow's et al.'s model and this probably explains the discrepancy between the predicted and observed robustness of the network (and, in extended form, of the overall organization of the stages). The discrepancy can further be explained by the crucial importance of concentration differences (of gene products) for patterning, which increases the sensitivity of the system to changes even when striped patterns are still generated. Why has more robustness not evolved? Perhaps, within these stages the total number of interactions involved in morphogenetic patterning is too limited to organize the pattern in an independent, modular way allowing greater robustness.

Even if the segmented gene network were robust, it would not provide long-term conservation for two reasons. Firstly, the organizer function of the segment polarity genes implies large consequences for small changes. This makes it almost impossible to avoid phenotypic effects. Secondly, robustness achieved during periods of ecological stasis, loses its effectiveness in periods with strong environmental changes and directional selection. Drift during episodes of stabilizing selection accumulates hidden genetic variation, which enables fast evolutionary change as soon as selection becomes directional.

The severe phenotypic effects of most investigated mutations indicate that there is no absence of genetic variation with phenotypic effects. Hence, strong stabilizing selection appears to be the major force in the conservation of the extended and segmented germband stages. The documented pleiotropic effects of mutations of these stages are in agreement with the hypotheses of Sander (7) and Raff (10) that negative pleiotropic effects of mutational changes resulting from global interactions are constraining evolutionary change. A considerable part of the pleiotropic effects is due to cascading interactions, indicating a low effective modularity (Box 1). It thus appears that low effective modularity constrains evolutionary change. 


\section{References}

1. Patel, N.H. et al. (1992) Changing the role of even-skipped during the evolution of insect pattern formation. Nature 357, 339-342

2. Holland L.Z. (2000) Body-plan evolution in the bilateria: early antero-posterior patterning and the deuterostome-protostome dichotomy. Cur. Opin. Genet. Devel. 10: $434-442$

3. Damen, W. G. M. et al. (2000). Expression patterns of hairy, even-skipped, and runt in the spider Cupiennius salei imply that these genes were segmentation genes in a basal arthropod. Proc. Natl. Acad. Sci. U.S.A. 97, 4515-4519

4. French, V. (2001) Insect segmentation: genes, stripes and segments in 'Hoppers'. Cur. Biol. 11, R910-913

5. Grbić, M (2000) Alien wasps and evolution of development. BioEssays 22, 920-932

6. Anderson, D.T. (1973) Embrology and Phylogeny in annelids and Arthropods. Oxford: Pergamon

7. Sander, K. (1983) The evolution of patterning mechanisms: gelanings from insect embryogenesis and spermatogenesis. In Development and Evolution (Goodwin, B.C. et al., eds), pp. 137-154, Cambridge University Press

8. Akam, M. (1987) The molecular basis for metameric pattern in the Drosophila embryo. Development 101, 1-22

9. McGinnis, W. and Krumlauf, R. (1992) Homeobox genes and axial patterning. Cell $68,283-302$

10. Raff, R.A. (1996) The shape of Life, University of Chicago Press

11. Hadorn, E. (1961) Developmental genetics and lethal factors. Methuen and co LTD, London; John Wiley and Sons, New York

12. Wright T.R. (1970) The genetics of embryogenesis in Drosophila. Adv. Genet. 15, 261-395

13. Von Dassow, G. and Munro E.M. (1999) Modularity in animal development and evolution: elements of a conceptual framework for EvoDevo. J. exp. Zool. (Mol. Dev. Evol.) 285, 307-325

14. on Dassow, G. et al. (2000) The segment polarity network is a robust development module. Nature 406, 188-192

15. Kacser, H. and Burns, J.A. (1981) The molecular basis of dominance. Genetics 97 , 639-666

16. Wagner, A. (2000) Robustness against mutations in genetic networks of yeast. Nature Genet. 24, 355-361

17. Müller, H.-A. J. et al. (1999) Wingless signaling in the Drosophila embryo: zygotic requirements and the role of the frizzled genes. Development 126, 577-586

18. Bateson, W. (1894) Materials for the study of variation, Macmillan. 
19. Eker, R. (1929) The recessive mutant engrailed in Drosophila melanogaster. Hereditas 12, 221-222

20. Tiniakov, G.G. and Terentieva , E.L. (1933) cubitus interruptus, a new genvariation of the fourth chromosome of Drosophila melanogaster. Genetics 18, 117-120

21. Bridges C.B. and Morgan, T.H. (1923) The third-chromosome group of mutant characters of Drosophila melanogaster. Carnegie Inst. Washington Publ. No. 327, 1251

22. Morgan, Th. et al. (1925). The genetics of Drosophila melanogaster. Bibliog. Genet. 2: $1-262$

23. Bejsovec, A. and Wieschaus, E. (1993) Segment polarity gene interactions modulate epidermal patterning in Drosophila embryos. Development 119, 501-517

24. Theisen, H. et al. (1994) Dishevelled is required during wingless signaling to establish both cel polarity and cell identity. Development 120, 347-360

25. Fujioka, M. et al. (1995) Early even-skipped stripes act as morphogenetic gradients at the single cell level to establish engrailed expression. Development 121, 43714382

26. Lawrence, P.A. and Pick, L. (1998) How does the fushi tarazu gene activate engrailed in the Drosophila embryo. Devel. Genet. 23, 28-34

27. Dierick, H.A. and Bejsovec, A. (1998) Functional analysis of Wingless reveals a link between intercellular ligand transport and dorsal-cell-specific signaling. Development 125, 4729-4738

28. McEwen, D.G. et al. (2000) The canonical Wg and JNK signaling cascades collaborate to promote both dorsal closure and ventral patterning. Development 127 , 3607-3617

29. Zeng, W. et al. (2000) Naked cuticle encodes an inducible antagonist of Wnt signalling. Nature 403, 789-795

30. Couso, J.P. and Martinez-Arias, A. (1994) Notch is required for wingless signalling in the epidermis of Drosophila. Cell 79, 259-272

31. Wesley, C.S. (1999) Notch and Wingless Regulate Expression of Cuticle Patterning Genes. Mol. Cel. Biol. 19, 5743-5758

32. Michelli, C.A. et al. (2002) rasp, a putative transmembrane acyltransferase, is required for Hedgehog signaling. Development 129, 843-851

33. Wu, X. et al. (1995). Heart development in Drosophila requires the segment polarity gene wingless. Dev. Biol. 169, 619-628

34. Simonds, A. J. et al. (1995) Distinguishable functions for engrailed and invected in anterior-posterior patterning in the Drosophila wing. Nature 376: 424-427

35. Gallet A, et al.(2000) Cubitus interruptus-independent transduction of the Hedgehog signal in Drosophila. Development 127, 5509-5522

36. Klingensmith, J., et al. (1989) The segment polarity phenotype of Drosophila involves different tendencies toward transformation and cell death. Devel. Biol. 134, $130-145$ 
37. Cohen, S.M. (1990) Sepcification of limb development in the Drosophila embryo by positional cues from segmentation genes. Nature 343, 173-177

38. Peifer et al. (1991) wingless signal and Zeste-white 3 kinase trigger opposing changes in the intracellular distribution of Armadillo. Development 120, 369-380

39. Sato, A. et al. (1999) Dfrizzled-3, a new Drosophila Wnt receptor, acting as an attenuator of Wingless signaling in wingless hypomorphic mutants. Development $126,4421-4430$

40. Couso, J.P.et al. (1994) The wingless signalling pathway and the patterning of the wing margin in Drosophila. Development 120, 621-636

41. Struhl, G. and Basler, K. (1993) Organizing activity of wingless protein in Drosophila. Cell 72, 527-540

42. Struhl et al. (1997) Hedgehog organizes the pattern and polarity of epidermal cells in the Drosophila abdomen. Development 124, 2143-2154

43. Sanson B. et al. (1999) Engrailed and Hedgehog make the range of Wingless asymmetric in Drosophila embryos. Cell 98, 207, 216

44. Duman-Scheel, M. et al. (1997) Genetic separation of the neural and cuticular patterning functions of gooseberry. Development 124, 2855-2865

45. Cox, R.T. et al. (2000) A screen for mutations that suppress the phenotype of Drosophila armadillo, the $\beta$-catenin homolog. Genetics $155,1725-1740$

46. Wakimoto, B.T. and T.C. Kaufman, (1981) Analysis of larval segmentation in lethal genotypes associated with Antennapedia gene complex in Drosophila melanogaster. Devel. Biol. 81, 51-64

47. Kornberg, T. (1981) Engrailed: A gene controlling compartment and segment formation in Drosophila. Proc. Natl. Acad. Sci. USA 78, 1095-1099

48. Sharma, R.P. and V.L. Chopra, V.L. (1976) Effect of the Wingless ( $\mathrm{wg}^{l}$ ) mutation on wing and haltere development in Drosophila melanogaster. Devel. Biol. 48, 461465

49. Van den Heuvel, M. et al. (1989) Distribution of the wingless gene product in Drosophila Embryos: A protein involved in cell-cell communication. Cell 59, 739749

50. Sanson, B. (2001) Generating patterns from fields of cells - Examples from Drosophila segmentation. EMBO Rep. 2, 1083-1088

51. Lawrence, P.A., Casal, J. and Struhl, G. (1999) Hegehog and engrailed: pattern formation and polarity in the Drosophila abdomen. Development 126, 2431-2439

52. Charlesworth, B., Lande, R. and M. Slatkin, (1982) A neo-darwinian commentary on macroevolution. Evolution 36, 474-498

53. Wagner, A. (1996) Does evolutionary plasticity evolve? Evolution 50: 1008-1023

54. Wagner, G.P. (1997) A population genetic theory of canalization. Evolution 51, 329347 
55. Gibson, G. and G. P. Wagner (2000) Canalization in evolutionary genetics: a stabilizing theory? BioEssays 22, 372-380.

56. Schuster, P. Fontana, W., Stadler, P.F. and Hofacker, I.L. (1994). From sequences to shapes and back. A case study in RNA secondary structures. Proc.Roy.Soc.Lond.B $255,279-284$

57. Stadler B.M.R., Stadler P.F., Wagner G.P., Fontana W. (2001) The topology of the possible: Formal spaces underlying patterns of evolutionary change. J. theor. Biol. 213, 241-274

58. Lande, R. et al. (1994) High inbreeding depression, selective interference among loci, and the threshold selfing rate for purging recessive lethal mutations. Evolution 48, 965-978

59. Galis, F. and Metz , J.A.J. (2001) Testing the vulnerability of the phylotypic stage: on modularity and evolutionary conservation. J. exp. Zool. (Mol. Dev. Evol.) 291, 195-204 